\title{
Inicio en el consumo de alcohol y tabaco y transición a otras drogas en estudiantes de Morelos, México
}

Magdalena Herrera-Vázquez, M en $C$, (1) Fernando A W agner, Ph D, (2) Eduardo Velasco-Mondragón, Ph D, ${ }^{(3)}$ Guilherme Borges, PhD, ${ }^{(4,5)}$ Eduardo Lazcano-Ponce, Dr en $C^{\left({ }^{(3)}\right.}$

Herrera-Vázquez M,Wagner FA,Velasco-Mondragón E, Borges G, Lazcano-Ponce E. Inicio en el consumo de alcohol y tabaco y transición a otras drogas en estudiantes de Morelos, México. Salud Publica Mex 2004;46:132-140. El texto completo en inglés de este artículo está disponible en: http://www.insp.mx/salud/index.html

\section{Resumen}

Objetivo. Estimar la probabilidad acumulada de ocurrencia del primer uso de alcohol y tabaco, y el riesgo de transitar hacia el uso inicial de otras sustancias (marihuana, cocaína y heroína, entre otras) en estudiantes ado lescentes y adultos jóvenes de Morelos, México. Material y métodos. Se llevó a cabo un estudio transversal en el ciclo escolar 19981999, en el estado de Morelos, en una muestra probabilística de estudiantes de entre 11 a 24 años de edad ( $n=13$ 293) a quienes se distribuyó un cuestionario auto-aplicable validado. Los datos se restructuraron para construir una cohorte sintética que se estudió con métodos de sobrevida y unidades discretas de tiempo. Se obtuvieron riesgos relativos y sus inter valos de confianza de $95 \%$, con modelos multivariados de regresión de Cox. Resultados. Sesenta por ciento de los varones iniciaron el uso de alcohol en promedio a los 17 y el uso de tabaco a los 18 años de edad. Las mujeres iniciaron el uso de alcohol y tabaco un año despues que los hombres. El uso de otras drogas ocurrió a los 19 años de edad en promedio en $5 \%$ de las mujeres y $13 \%$ de los varones. N ueve de cada 100 estudiantes que consumieron drogas ilegales iniciaron directamente sin antes ha-
Herrera-Vázquez M,Wagner FA, Velasco-Mondragón E, Borges G, Lazcano-Ponce E.

Onset of alcohol and tobacco use and transition to other drug use among students from Morelos, Mexico. Salud Publica Mex 2004;46:132-140.

The English version of this paper

is available at: http://www.insp. mx/salud/index.html

El presente trabajo se realizó bajo el financiamiento de la Fundación Bristol Myers Squibb de N ueva York, EUA.Asimismo, el Consejo N acional de Ciencia y Tecnología de México (N úm 34487-M) y el Instituto N acional de Salud Pública de México, financiaron parcialmente el análisis de la información. El doctor W agner recibió apoyo del $\mathrm{N}$ ational Institute on DrugAbuse de EUA (5U24-DA12390-02).

(1) Instituto de Seguridad y Servicios Sociales de losTrabajadores del Estado, Delegación Estatal Puebla, Puebla, Puebla, México.

(2) Drug A buse Research Program, Public Health Program. Morgan State University. Baltimore, Maryland, Estados Unidos de América (EUA).

(3) Centro de Investigaciones en Salud Poblacional. Instituto N acional de Salud Pública. Cuernavaca, Morelos, México.

(4) Universidad Autónoma Metropolitana-X ochimilco, México, DF, México.

(5) Instituto $N$ acional de Psiquiatría. México, DF, México.

Fecha de recibido: 20 de marzo de 2003 - Fecha de aprobado: 17 de octubre de 2003

Solicitud de sobretiros: Dr. Eduardo Lazcano Ponce. Director de Enfermedades Crónicas. Centro de Investigación en Salud Poblacional. Instituto N acional de Salud Pública. Avenida Universidad 655, colonia Santa María A huacatitlán, 62508 Cuernavaca, Morelos, México. Correo electrónico: elazcano@ correo.insp.mx 
ber usado alcohol ni tabaco. En general, los estudiantes usuarios de alcohol o tabaco, 0 ambos, presentaron mayor riesgo de iniciar el uso de otras drogas que los no usuarios $(R R=6.72$; IC 95\%=4.13-10.93). Conclusiones Son claras las implicaciones potenciales del presente estudio al considerar que, junto con intervenciones encaminadas a disminuir el consumo de drogas, resulta igualmente importante retardar la edad de inicio de alcohol y tabaco por sexo, ya que mediante un nuevo enfoque brinda evidencia epidemiológica que relaciona el uso de estas sustancias con el uso posterior de otras drogas en estudiantes mexicanos. El texto completo en inglés de este artículo está disponible en: http://www.insp.mx/salud/index.html

Palabras clave: abuso de sustancias; edad de inicio; adolescencia; estudiantes; México alcohol or tobacco users were at greater risk of starting drug use than nonusers ( $R R=6.72 ; 95 \%$ confidence interval $[C l]=4.13-10.93)$. Conclusions Study findings suggest that interventions to decrease drug abuse should go to gether with efforts to delay initiation of alcohol and tobacco use. The innovative method used in this study yields epidemiolo gic evidence relating early use of alco hol and to bacco with initiation of illegal drugs in Mexican students. The English version of this paper is available at: http://www.insp.mx/salud/index.html

Key words: substance abuse; age of on set; adolescence students; Mexico
E 1 uso de sustancias psicoactivas es motivo de preocupación por provocar problemas de salud pública de gran impacto mundial. El incremento de la población vulnerable (jóvenes), de los factores de riesgo derivados de las transiciones sociales y de la disponibilidad de las drogas, se han propuesto como factores que condicionan el aumento en el número de nuevos usuarios y la disminución en su edad de inicio. ${ }^{1}$

Definida como la etapa entre la niñez y la edad adulta, la adolescencia (de los 10 a los 19 años de edad) constituye una de las etapas más vulnerables del ser humano para desarrollar costumbres y hábitos de riesgo para su salud, entre los que destacan el alcoholismo, el tabaquismo y la farmacodependencia. ${ }^{2-4} \mathrm{La}$ evidencia epidemiológica nacional e internacional reconoce que el uso ocasional o continuo de alcohol y tabaco, solos o combinados, permanece obstinadamente común entre la gente joven, ${ }^{5-12}$ con mayores prevalencias de uso en el sexo masculino, ${ }^{2,13,14}$ con mayor número de usuarios de alcohol que de tabaco y mayor preferencia por el alcohol como droga de inicio en los estudiantes de 12 a 19 años de edad;12,15-18 así también, muestra que la edad de inicio es una variable fuertemente asociada al consumo de drogas. ${ }^{19-22}$

Por otro lado, los modelos de la "piedra angular" ("stepping-stone model") y de "puerta de entrada o portal" ("gateway model") ofrecieron las primeras observaciones epidemiológicas acerca del vínculo entre el uso de alcohol o tabaco y un exceso de riesgo para iniciar el uso de otras drogas, como marihuana y cocaína. ${ }^{23-27}$ En estos modelos, el uso inicial de alcohol o tabaco se asocia con un riesgo mayor de iniciar el uso de marihuana, lo que a su vez incrementa el riesgo de usar cocaína y otras drogas..$^{5,28-30}$
Debido a su prevalencia y a la forma en que los mexicanos consumen alcohol (con baja frecuencia pero en grandes cantidades y con marcada tendencia a la embriaguez), el consumo de alcohol representa un grave problema de salud pública en México ${ }^{12,13,15,31,32}$ que se aúna al impacto del tabaco y, en menor escala, de la marihuana, la cocaína y otras drogas de consumo menos prevalente. ${ }^{12,33-38}$ De esta manera, se hace evidente en México la necesidad de ahondar en las investigaciones con relación a las edades de inicio y transición en el uso temprano de alcohol y tabaco, para determinar su papel en la transición al uso posterior de otras drogas como marihuana y cocaína, lo que puede constituir un blanco importante de los esfuerzos de prevención, pues la ocurrencia del evento en el tiempo ayuda a entender el valor preventivo de las acciones. ${ }^{1,4}$ En nuestro país, se han realizado múltiples estudios transversales que exploran, entre otros tópicos, el inicio en el uso de drogas, $;, 7,13,15,17,34,35,37,39,40$ sin embargo, no se reportan investigaciones en México ni en Latinoamérica que incorporen en su metodología el análisis de sobrevida para estimar la edad de inicio en el consumo de alcohol, tabaco, marihuana y cocaína, ni estudios que consideren sus transiciones temporales en una encuesta poblacional transversal.

En atención a los elementos planteados anteriormente, el presente estudio busca estimar la asociación del uso temprano de alcohol o tabaco, con la progresión hacia el consumo de otras drogas como marihuana y cocaína entre estudiantes de enseñanza básica secundaria media y superior del estado de Morelos. Los hallazgos de este estudio buscan aportar información para la formulación de políticas dirigidas a restringir la disponibilidad de bebidas alcohólicas y 
cigarrillos de tabaco en los jóvenes, en cuanto a que se investiga si el uso temprano de alcohol y tabaco los predispone al inicio de drogas ilegales.

\section{Material y métodos}

Los datos para la presente investigación provienen del estudio Estilos de vida en adolescentes y adultos jóvenes y su relación con el desarrollo longitudinal de exposición a enfermedades crónicas, llevado a cabo en el estado de Morelos durante el ciclo escolar 1998-1999, por el Instituto Nacional de Salud Pública, en colaboración con las Secretarías de Salud y de Educación Pública locales. Dicho estudio ha sido descrito en detalle en otras publicaciones, ${ }^{41}$ por lo que en el presente artículo se ofrece una síntesis de sus características. Se trata de una encuesta transversal, efectuada en el ámbito escolar con representación estatal de las zonas de residencia urbana, semiurbana y rural, con un diseño de muestra aleatorio, polietápico y sistemático, donde la primera unidad de muestreo fueron las escuelas, todas con la misma probabilidad de selección. En total, se logró la participación de 13293 estudiantes (7 468 mujeres y 5825 varones) de entre 11 y 24 años de edad, a quienes se distribuyó un cuestionario autoaplicable. Encuestadores previamente entrenados se presentaron en cada grupo escolar para hacer contacto con los alumnos y explicarles los pormenores de la encuesta. Se puso especial cuidado en que los encuestadores supieran transmitir las instrucciones que garantizaran a los alumnos la confidencialidad y el absoluto anonimato de sus respuestas. Antes de repartir el cuestionario se obtuvo el consentimiento por escrito de los padres o tutores y la participación del adolescente se sujetó a su aceptación voluntaria. La tasa de respuesta obtenida fue de $98.6 \%{ }^{41}$

Para recabar información sobre uso de alcohol, tabaco y otras drogas como marihuana, cocaína, heroína, cemento y thinner, entre otras, se utilizó una versión adaptada del instrumento utilizado previamente en las encuestas de estudiantes del país, cuyo diseño está basado en el cuestionario para estudiantes aprobado por la Organización Mundial de la Salud (OMS), cuyas preguntas están ya estandarizadas y tienen validez y confiabilidad satisfactorias. ${ }^{12,15,42,43} \mathrm{El}$ cuestionario incluyó las secciones de uso de tabaco, alcohol y otras drogas, edad de inicio en el consumo de alcohol, tabaco y otras drogas, así como datos sociodemográficos en cuanto a la edad al momento de la encuesta, sexo, grado escolar, nivel educativo y nivel socioeconómico.

Se consideró el uso inicial de alcohol o tabaco para el análisis de la transición a otras drogas, ya que, en la mayoría de las versiones, el modelo "puerta de entrada" identifica el primer paso de la implicación con drogas en términos del uso de alcohol y tabaco, solos o combinados. ${ }^{23-26,30,44}$ Se construyó una variable para obtener la edad de la primera ocurrencia del uso de cualquiera de las dos drogas. En relación con otras drogas, el cuestionario considera la edad de inicio de manera global sin discriminar la edad por tipo de droga en una sola pregunta. Se definió como "no usuarios" a los estudiantes que nunca han consumido ninguna de las drogas investigadas, y por "usuarios", a quienes han consumido las drogas alguna vez en su vida. ${ }^{40,45}$ La variable de respuesta se construyó tomando en cuenta el tiempo que transcurrió desde el momento en que nació el sujeto de estudio o año de nacimiento (evento inicial $u$ origen) hasta que inició su primera experiencia en el consumo de alcohol, tabaco y otras drogas (eventos finales o resultados de interés). La variable de censura se definió por los estudiantes que no presentaron el evento de interés o evento final al momento de la encuesta. Entre las variables sociodemográficas, el tipo de residencia se estableció de acuerdo a la categorización municipal desarrollada por el Instituto Nacional de Estadística, Geografía e Informática, con base en el Censo Nacional de Población y Vivienda, que utiliza como indicadores el número de habitantes y nivel de marginación. ${ }^{46}$ Para el índice de nivel socioeconómico, siguiendo el procedimiento utilizado por Rabe-Hesketh ${ }^{47}$ y Jolliffe, ${ }^{48}$ se generó una variable ordinal del 1 al 10, mediante un análisis de componentes principales. ${ }^{41}$ Las variables utilizadas fueron: características de la vivienda (tipo de piso, presencia de agua potable y drenaje), ingreso familiar y disponibilidad de casa propia, auto, gas estacionario, refrigerador, teléfono, televisión a color y videocasetera. El índice se conformó en tres categorías ordinales agrupadas a partir de los terciles. Con base en una perspectiva del desarrollo que considera distintos procesos vitales a lo largo de los años, como transiciones biopsicológicas y educacionales (ingreso a educación básica secundaria, por ejemplo), la edad para el análisis del uso de alcohol o tabaco se dividió en cuatro categorías (11-14, 15, 16-18 y 19-21) de acuerdo a Anthony y Petronis, ${ }^{21}$ Wagner y Anthony, ${ }^{49}$ Wilcox y colaboradores $^{50}$ y Caraveo y colaboradores, ${ }^{51}$ excluyendo el grupo de 22 a 24 años de edad, pues además de tener muy pocas observaciones (188) se ha visto que después de los 20 años es poco común que los estudiantes inicien el uso de alcohol o tabaco. ${ }^{15,31,34,49,52-54}$ Del total de cuestionarios, 1772 se excluyeron del análisis por no proveer datos sobre la edad de inicio del uso de sustancias. La mayoría de ellos correspondían a estudiantes de entre 12 y 14 años de edad. 
Debido a que el tiempo en esta encuesta fue medido en una escala de intervalos anual y bianual, se utilizó el modelo de análisis de sobrevida sugerido por $\mathrm{Cox}^{55}$ cuando las unidades de tiempo son discretas, modelo discutido por Breslow y Day ${ }^{56}$ y recientemente empleado en el contexto del uso de drogas por Singer y Willet, ${ }^{57-59}$ Wilcox y colaboradore ${ }^{50}$ y Wagner y Anthony. ${ }^{53}$ Dicho modelo es una extensión del de riesgos proporcionales de Cox. El modelo de tiempo discreto retiene la función hazard multiplicativa del modelo de tiempo continuo e igualmente permite modelar el tiempo al evento, ajustando o estratificando por covariables, tomando en cuenta el periodo de reclutamiento de los sujetos, diferentes tiempos de ingreso al estudio y sesgos de temporalidad. La diferencia principal con respecto al modelo de riesgos proporcionales es que el análisis de sobrevida con tiempo discreto es capaz de asumir estadísticamente el hecho de que la medición del tiempo se ha realizado en unidades discretas de tiempo (por ejemplo años, en el presente estudio). En este estudio se ocupó el análisis de sobrevida en tiempo discreto para estimar la probabilidad acumulada de iniciar el consumo de alcohol, tabaco y otras drogas, como la proporción de estudiantes que inició su consumo hasta una edad determinada. ${ }^{7,49,56-58}$ Se aplicaron dos perspectivas de desarrollo: a) el tiempo se midió cronológicamente en años desde el nacimiento, y b) la escala de tiempo se colocó en años desde el primer uso para alcohol, tabaco y otras drogas.

Para examinar las transiciones del uso de alcohol o tabaco, o de ambos, al uso de otras sustancias, se estimó la probabilidad de iniciar el uso entre los estudiantes que reportaron su consumo por primera vez, por lo que para este análisis, el tamaño muestral fue menor (5 994 para alcohol o tabaco y 594 para otras drogas). El manejo y análisis de la información se realizó utilizando el paquete estadístico Stata $8.0^{60} \mathrm{e}$ incluyó estimaciones de probabilidad acumulada de la transición desde el primer uso de una droga al primer uso de la otra. Para facilitar la comprensión de la forma en que la probabilidad cambia en función del tiempo, las estimaciones se presentan gráficamente. Los modelos de regresión de Cox en tiempo discreto, al ajustarse por las covariables en estudio, permitieron estimar el peligro o riesgo relativo (Relative Hazard) de iniciar el uso de alcohol, tabaco y otras sustancias, puesto que no se tenía historia previa de uso, así como el riesgo de empezar a usar drogas como marihuana y cocaína con relación al uso previo de alcohol, tabaco, o ambos. El inicio del uso de alcohol, tabaco y otras drogas se codificó como variante en el tiempo (" 0 " hasta el momento del evento y " 1 " de allí en adelante), mien- tras el sexo, la edad al momento de la entrevista, el nivel educativo, el nivel socioeconómico y el tipo de residencia no variaron en el tiempo. Los análisis de regresión se estratificaron por escuela para controlar el efecto de conglomerado sobre la estimación de la varianza y restringir la confusión potencial por las características del entorno escolar, como disponibilidad de drogas, presencia policiaca y nivel de desorganización comunitaria. ${ }^{61}$

\section{Resultados}

El cuadro I describe las variables de uso de alcohol o tabaco y de otras drogas. En él se observa que, a los 15 años de edad, 50\% de los estudiantes ya había iniciado el consumo de alcohol o tabaco. Asimismo, se puede observar que haber usado alcohol o tabaco previamente se asocia con mayores posibilidades de iniciar el uso de otras drogas. Aproximadamente $90 \%$ de quienes usaron alcohol o tabaco nunca iniciaron el uso de otras sustancias; por otro lado, siete de cada 1000 estudiantes iniciaron directamente el uso de otras drogas sin que hubiesen consumido alcohol ni tabaco. En general, se encontró que los varones, los estudiantes de preparatoria y universidad, aquellos de entre 19 y 21 años de edad, los que provienen del nivel socioeconómico alto y aquellos que viven en áreas urbanas, mostraron mayores posibilidades de iniciar el uso de todas las drogas estudiadas, comparados con las mujeres, los estudiantes de secundaria, aquellos de entre 11 y 14 años de edad, los que provienen del nivel socioeconómico bajo y los que viven en el área rural, respectivamente.

La figura 1 ofrece una representación gráfica de las probabilidades acumuladas de tiempo al inicio del uso de alcohol, tabaco y otras drogas por edad y sexo. Los hombres mostraron mayores porcentajes de inicio en el uso en todas las edades estudiadas, comparados con las mujeres. A la edad de 17 años, $60 \%$ de los varones había iniciado ya el uso de alcohol y $60 \%$ inició el uso de tabaco a los 18. Sesenta por ciento de las mujeres había empezado a usar alcohol a los 18 años, mientras que $40 \%$ había iniciado el uso de tabaco a los 19 años. Entre mujeres, la probabilidad acumulada estimada para iniciar el uso de otras drogas fue de $1 \%$ a los 14 años y aumentó a 5\% a los 19 años; después de esta edad, no hubo incrementos. Esta probabilidad acumulada entre varones, fue de $1 \%$ a los 12 años y de $15 \%$ a los 20 años. La evidencia fue en el sentido de la hipótesis inicial, y mostró que existen diferencias significativas en el tiempo al uso entre hombres y mujeres para todas las drogas estudiadas, por lo que los análisis subsecuentes se estratificaron por sexo (alcohol: 


\section{Cuadro I \\ Características seleccionadas de los estudiantes de enseñanza básica media Y SUPERIOR INCLUIDOS EN EL ANÁLISIS DEL USO INICIAL DE ALCOHOL, TABACO Y OTRAS DROGAS.* Estado de Morelos, México, 1998-1999}

Característica

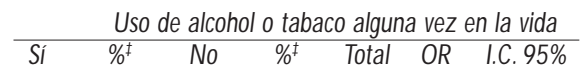

Uso de otras drogas alguna vez en la vida

Uso previo de alcohol o tabaco

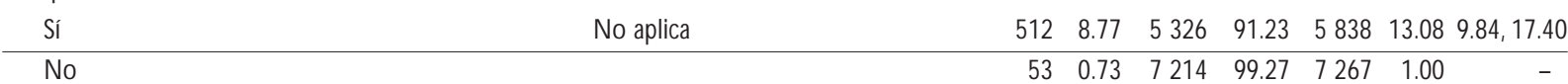

Edad actual

\begin{tabular}{|c|c|c|c|c|c|c|c|c|c|c|c|c|c|c|}
\hline $19-21$ & 466 & 75.65 & 150 & 24.35 & 616 & 4.82 & $3.99,5.82$ & 58 & 9.42 & 558 & 90.58 & 616 & 2.66 & $1.99,3.55$ \\
\hline $16-18$ & 1125 & 59.87 & 754 & 40.13 & 1879 & 2.32 & $2.09,2.56$ & 105 & 5.59 & 1774 & 94.41 & 1879 & 1.51 & $1.21,1.89$ \\
\hline 15 & 418 & 49.94 & 419 & 50.06 & 837 & 1.55 & $1.34,1.78$ & 34 & 4.06 & 803 & 95.94 & 837 & 1.08 & $0.76,1.55$ \\
\hline 11-14 & 3829 & 39.18 & 5944 & 60.82 & 9773 & 1.00 & - & 368 & 3.77 & 9405 & 96.23 & 9773 & 1.00 & \\
\hline
\end{tabular}

Sexo

\begin{tabular}{lcccccccccccccc} 
Hombre & 3086 & 53.90 & 2639 & 46.10 & 5725 & 1.97 & $1.83,2.11$ & 414 & 7.23 & 5311 & 92.77 & 5725 & 3.73 & $3.09,4.51$ \\
\hline Mujer & 2752 & 37.29 & 4628 & 62.71 & 7380 & 1.00 & - & 151 & 2.05 & 7229 & 97.95 & 7380 & 1.00 & -
\end{tabular}

Nivel socioeconómico

\begin{tabular}{|c|c|c|c|c|c|c|c|c|c|c|c|c|c|c|}
\hline Alto & 1799 & 60.37 & 1181 & 39.63 & 2980 & 2.69 & $2.43,2.98$ & 188 & 6.31 & 2792 & 93.69 & 2980 & 2.18 & $1.70,2.80$ \\
\hline Medio & 2843 & 41.72 & 3972 & 58.28 & 6815 & 1.26 & $1.16,1.38$ & 278 & 4.08 & 6537 & 95.92 & 6815 & 1.38 & $1.09,1.74$ \\
\hline Bajo & 1196 & 36.13 & 2114 & 63.87 & 3310 & 1.00 & - & 99 & 2.99 & 3211 & 97.01 & 3310 & 1.00 & \\
\hline
\end{tabular}

$N$ ivel educativo

\begin{tabular}{|c|c|c|c|c|c|c|c|c|c|c|c|c|c|}
\hline ersidad & 1553 & 64.25 & 864 & 35.75 & 2417 & 2.69 & $2.45,2.94$ & 159 & 6.58 & 2258 & 93.42 & 417 & 1.78 \\
\hline aria & 4285 & 40.09 & 6403 & 59.9 & 10688 & 1.00 & - & 406 & 3.80 & 10282 & 96.20 & 0688 & 1.00 \\
\hline
\end{tabular}

Tipo de residencia

\begin{tabular}{|c|c|c|c|c|c|c|c|c|c|c|c|c|c|c|}
\hline Urbana & 2611 & 56.67 & 1996 & 43.33 & 4607 & 2.45 & $2.26,2.66$ & 290 & 6.29 & 4317 & 93.71 & 4607 & 2.22 & $1.82,2.71$ \\
\hline Semiurbana & 1399 & 43.10 & 1847 & 56.90 & 3246 & 1.42 & $1.30,1.55$ & 121 & 3.73 & 3125 & 96.27 & 3246 & 1.28 & $1.01,1.63$ \\
\hline Rura & 1828 & 34.8 & 3424 & 65.19 & 5252 & 1.00 & - & 154 & 2.93 & 5098 & 97.07 & 5252 & 1.00 & \\
\hline
\end{tabular}

* Porcentajes del renglón

₹ Uso de alcohol y tabaco, solos o combinados

$n=13105$

$\chi_{(1 \mathrm{df})}^{2}=168.33, p<0.0001$; tabaco: $\chi_{(1 \mathrm{df})}^{2}=389.11, p<0.0001$; otras drogas: $\left.\chi_{(1 \mathrm{df})}^{2}=79.91, p<0.0001\right)$.

La figura 2 ofrece estimaciones de la probabilidad acumulada de transitar al uso de marihuana, cocaína y otras drogas entre usuarios y no usuarios de alcohol o tabaco, o de ambos, (27 821 años persona). Los usuarios de alcohol o tabaco tuvieron más posibilidades de iniciar el uso de otras drogas que los no usuarios $(p<0.001)$. En general, al comparar su probabilidad acumulada se observó que hasta la edad de 16 años, 1\% de los no usuarios de alcohol o tabaco había iniciado el uso de otras drogas, en contraste con quienes ya ha- bían usado uno u otro, o ambos, entre quienes uno de cada 10 ya había iniciado el uso de otras drogas antes de tener 17 años. La probabilidad acumulada de uso de otras drogas entre quienes consumieron alcohol o tabaco, o ambas sustancias, continúa en ascenso en años subsiguientes.

El cuadro II muestra los modelos de Cox en tiempo discreto, ajustados por diversas variables, para el inicio en el uso de drogas. De acuerdo a las hipótesis del presente trabajo, en este modelo se encontró una interacción multiplicativa entre el sexo y el uso de alcohol o de tabaco; de esta manera, se observó que los 


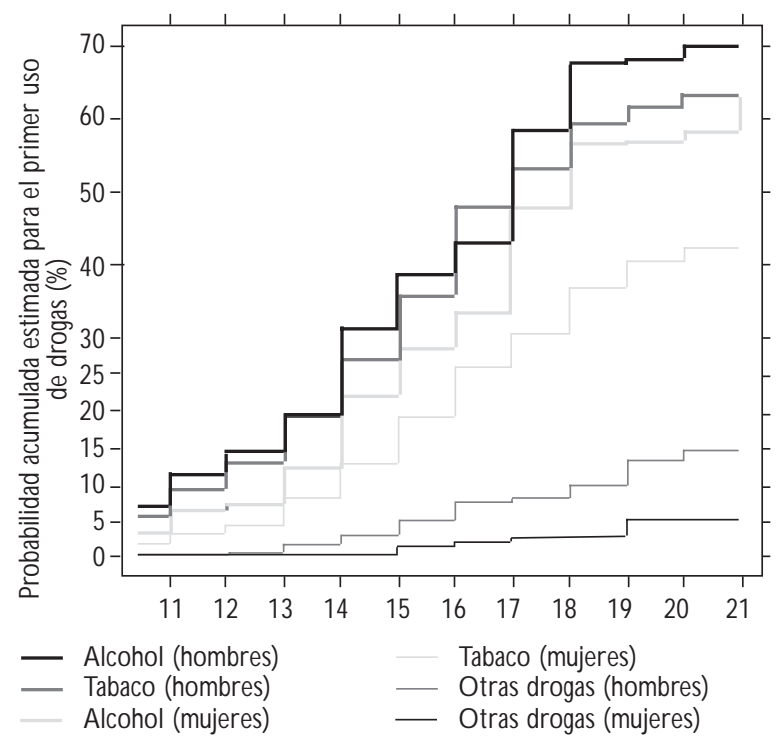

Figura 1. Probabilidad acumulada estimada para el PRIMER USO DE ALCOHOL, TABACO Y OTRAS DROGAS, POR SEXO, EN ESTUdIANTES DE ENTRE 11 Y 21 AÑoS DE EDAD. Estado de Morelos, México, 1998-1999

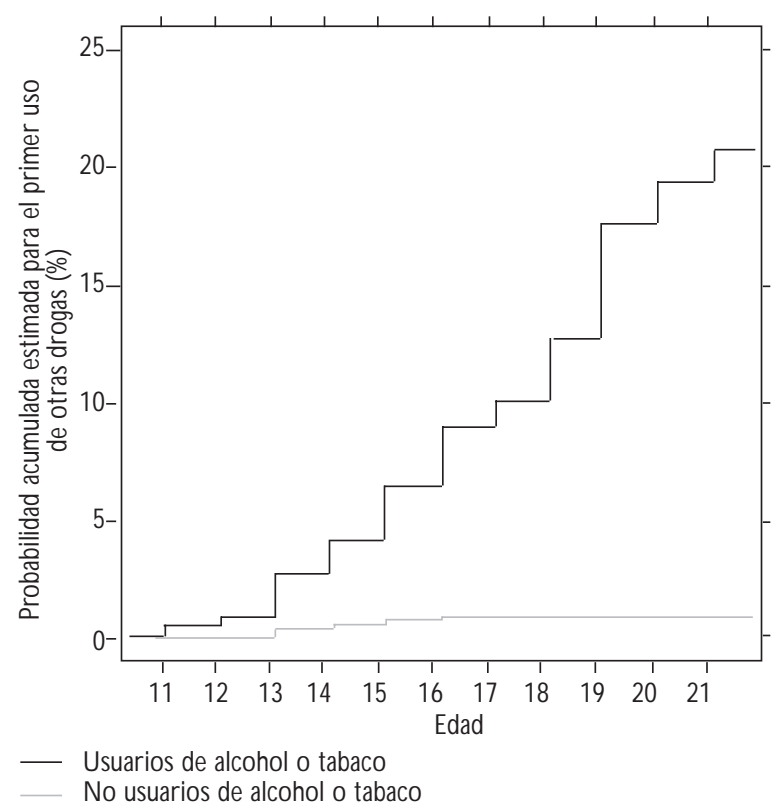

Figura 2. Probabilidad acumulada estimada para TRANSITAR AL USO INICIAL DE OTRAS DROGAS ENTRE USUARIOS DE ALCOHOL O TABACO VERSUS NO USUARIOS DE ALCOHOL O TABACO, PARA JÓVENES ENTRE 11 Y 21 AÑOS de edad. Estado de Morelos, México, 1998-1999

salud pública de méxico / vol.46, no.2, marzo-abril de 2004

\section{Cuadro II}

Riesgo Relativo para el Uso INICIAL de otras DROGAS EN ESTUDIANTES DE ENSEÑANZA MEDIA Y SUPERIOR. Estado de Morelos, México, 1998-1999* $R^{*} \quad$ IC $95 \%$

Sexo e historia de uso de alcohol o tabaco

\begin{tabular}{llll} 
Hombres usuarios de alcohol o tabaco & 9.16 & $5.16,16.25$ \\
\hline Mujeres usuarias de alcohol o tabaco & 3.44 & $1.85,6.39$ \\
\hline Mujeres no usuarias de alcohol o tabaco & 0.12 & 0.03, & 0.41 \\
\hline $\begin{array}{l}\text { Hombres no usuarios de alcohol o tabaco } \\
\text { (categoría de referencia) }\end{array}$ & 1.00 & -
\end{tabular}

Edad en grupos

\begin{tabular}{lll} 
19-21 años & 0.80 & $0.13,4.71$ \\
\hline $16-18$ años & 0.84 & $0.15,4.53$ \\
\hline 15 años & 0.97 & $0.51,1.82$ \\
\hline $11-14$ años (categoría de referencia) & 1.00 & -
\end{tabular}

Nivel socioeconómico

\begin{tabular}{|c|c|c|}
\hline Medio & 1.07 & $0.69,1.67$ \\
\hline Alto & 0.73 & $0.43,1.24$ \\
\hline Bajo (categoría de referencia) & 1.00 & \\
\hline
\end{tabular}

Tipo de residencia

\begin{tabular}{|c|c|c|}
\hline Semi-urbana & 0.56 & $0.18,1.72$ \\
\hline Urbana & 0.44 & $0.17,1.11$ \\
\hline Rural (categoría de referencia) & 1.00 & - \\
\hline
\end{tabular}

varones usuarios de alcohol o de tabaco presentaron más riesgo de iniciar el uso de otras drogas en comparación con los varones no usuarios ( $R R=9.16$, IC $95 \%$ $=5.16-16.25)$. Las mujeres usuarias presentaron tres veces mayor riesgo de empezar a usar otras drogas que los varones no usuarios ( $R R=3.44$, IC $95 \%=1.85-6.39)$. Por otro lado, las mujeres que no han usado alcohol ni tabaco tuvieron menor riesgo que los hombres en estas mismas condiciones ( $R R=0.12$, IC $95 \%=0.03-0.41$ ). Estos estimados son ajustados para posibles diferencias en cuanto a edad, nivel socioeconómico, y tipo de residencia, intervalo de riesgo, y características medidas y no medidas de escuela.

\section{Discusión}

Los principales hallazgos de este estudio pueden resumirse de la siguiente manera: a) la prevalencia general para el inicio del uso de alcohol entre los varones fue de $60 \%$ a los 17 años y para el uso de tabaco a los 
18 años de edad. Entre las mujeres, 60\% había empezado a usar alcohol a los 18 años de edad y 40\% había iniciado uso de tabaco a los 19 años. El primer uso de otras drogas como marihuana, cocaína, o la heroína había ocurrido en 5\% de las mujeres a los 19 años o antes, mientras que la proporción fue de $13 \%$ para los varones; $b$ ) éstos tienen casi dos veces más riesgo que las mujeres de iniciar el uso de alcohol o de tabaco y aquellos que ya lo han iniciado presentan nueve veces más riesgo para el inicio en el uso de otras drogas en comparación con los varones no usuarios; c) las mujeres usuarias presentaron tres veces mayor riesgo de empezar a usar otras drogas que los varones no usuarios; d) las mujeres que no han usado alcohol ni tabaco tienen mucho menor riesgo de usar otras drogas que los hombres sin antecedente de estas sustancias.

$\mathrm{Al}$ igual que en investigaciones previas, el presente estudio ubica al consumo de alcohol o tabaco como un importante antecedente del inicio en el consumo de otras sustancias, ya que se observa que la proporción de usuarios aumenta con la edad y documenta una vez más un exceso de riesgo asociado al sexo masculino. $2,5,14,16,17,62$ También es consistente con numerosos estudios sobre la teoría "puerta de entrada a las drogas" basados en observaciones de la progresión temporal en cantidad y frecuencia, que por otra parte, indican que el uso de alcohol o de tabaco en la adolescencia temprana (12-14 años) sitúa al individuo en gran riesgo de uso posterior e incrementa sus posibilidades de iniciar el consumo de otras drogas como marihuana y cocaína de los 18 a los 24 años de edad..$^{5,23-30}$ Las prevalencias halladas para el uso de alcohol, tabaco y drogas son acordes con las reportadas en diversos estudios mexicanos. ${ }^{2,11,15,33,40,62} \mathrm{Al}$ respecto, cabe señalar que los modelos de la "piedra angular" y "puerta de entrada" han resultado útiles para describir, pero no explicar, etapas o estratos de involucramiento en el consumo de drogas, y con el fin de aumentar la capacidad explicativa de esos modelos es preciso incorporar elementos intermedios que, siendo condición necesaria, vinculen una etapa de evolución en el consumo de drogas con la siguiente. Anthony, Van Etten y colaboradores han desarrollado estudios acerca de un posible mecanismo para comprender el proceso de involucramiento en el uso de drogas: la exposición a oportunidades para usarlas. ${ }^{49,63-66}$ La evidencia de estos autores señala que el exceso de ocurrencia en el uso de drogas entre hombres se remonta a diferencias en la exposición a oportunidades para usar drogas entre sexos, y más recientemente ofrecen evidencia en favor de la idea de que los usuarios de alcohol o de tabaco tienen mayor riesgo de usar marihuana, cocaína y otras drogas precisamente porque en cada etapa de involu- cramiento tienen mayor exposición a oportunidades para usarlas que los no usuarios. ${ }^{49-50,53}$

Sin embargo, en consideración a las posibles implicaciones de estas observaciones, es preciso reconocer importantes limitaciones que afectan a estudios de este tipo. En primer lugar, considerar que la restricción del marco muestral a jóvenes estudiantes puede limitar la generalización de los resultados a la población de gente joven o a subgrupos poblacionales con altas tasas de uso, como los adolescentes que sólo trabajan o los que viven en las calles, así como los que estudian en escuelas privadas. ${ }^{2,13,22,33,38,67}$ Inclusive, el presente estudio puede tener limitaciones derivadas del análisis de la naturaleza sintético-retrospectiva de los datos, especialmente preparados para dilucidar secuencias o transiciones de eventos discretos. ${ }^{49,57}$ Otro cuestionamiento probable se refiere a la validez y confiabilidad de los cuestionarios de autorreporte y al hecho de que una parte importante de los alumnos entre 12 y 14 años de edad no contestó las preguntas relativas al primer uso de alcohol o tabaco y, por tanto, hubo que basar las estimaciones en los datos realmente existentes. Sin embargo, estos problemas son comunes en la mayoría de los estudios sobre el uso de sustancias en población joven, y afortunadamente existen trabajos que sugieren que la validez y confiabilidad de las mediciones de autorreporte sobre la edad de inicio del uso de sustancias es suficientemente buena ${ }^{43,68} \mathrm{y}$ satisface los requisitos de calidad para la mayoría de las aplicaciones epidemiológicas actuales. ${ }^{69}$

No obstante éstas y otras importantes limitaciones, una de las principales fortalezas de este estudio es que los datos derivan de una encuesta poblacional probabilística y el empleo de métodos estadísticos capaces de acomodar la estructura compleja de la muestra (diferente probabilidad de selección y efectos de conglomeración al interior de las unidades primarias de muestreo). Así, es de esperar que en el presente estudio ciertos sesgos de selección hayan sido menores que en estudios con poblaciones de pacientes o voluntarios.

Acaso una de las contribuciones más sustanciales del presente estudio es que sienta un precedente en la investigación mexicana sobre el inicio y la progresión en el uso de drogas mediante el empleo de técnicas para el análisis de sobrevida. El hecho es importante porque una parte sustantiva de los participantes aún no ha terminado de vivir a través del periodo de mayor riesgo tanto para el inicio como para el involucramiento en el uso de drogas, lo cual puede conducir a subestimaciones importantes en la probabilidad de inicio, como en cuanto a la fuerza de la asociación con diversos factores de riesgo. La evidencia presentada 
en el presente artículo confirma la necesidad de realizar diversos esfuerzos para prevenir y atender el uso de alcohol, tabaco y otras drogas en las escuelas de nuestro país.

\section{Agradecimientos}

A la Secretaría de Educación Pública y de Salud del Estado de Morelos que brindaron todas las facilidades para realizar este estudio.

\section{Referencias}

1. Sepúlveda-Amor J. La salud en México a la vuelta del siglo. D esafíos, instrumentos, respuestas. Cuernavaca, Morelos, México. Julio de 2000. 2. C aballero R, Madrigal-de León E, San Martín AH,Villaseñor A. El consumo de tabaco, alcohol y drogas ilegales en los adolescentes de diferentes estratos socioeconómicos de Guadalajara. Salud Mental 1999;22:1-8.

3. Eisner MD, Yelin EH, Katz PP, Shiboski SC, Henke J, Blanc PD. Predictors of cigarette smoking and smoking cessation among adults with asthma.Am I Public Health 2000:90:1307-1311.

4. 0 rganización Panamericana de la Salud. La Salud en las Américas. W ashington, DC: 0 PS; 1998. (Publicación Científica N 0. 569, vol. I). 5. Kosterman R, H awkins JD, Guo J, C atalano RF,A bbott RD.The dynamics of alcohol and marijuana initiation: Patterns and predictors of first use in adolescence. Am J Public Health 2000 March; 90:360-366. 6. Giovino GA, Henningfield JE,Tomar SL, Escobedo LG, Slade J. Epidemiology of tobacco use and dependence. Epidemiol Rev 1995;17: 48-65

7. Berenzon S,Villatoro J, Medina-Mora ME, Fleiz C, A Icántar E, N avarro C. El consumo de tabaco de la población estudiantil de la Ciudad de México. Salud Mental 1999; 22:20-25.

8. 0 rganización Panamericana de la Salud. La Salud en las A méricas. W ashington, DC: O PS; (Publicación Científica No 569 vol. II. ), 1998:586. 9. Secretaría de Salud. Encuesta N acional de Adicciones 1993. Alcohol. México, D F: SSA-Dirección General de Epidemiología; 1994.

10. Secretaría de Salud. Encuesta N acional de Adicciones 1993. Tabaco. México, DF: SSA-Dirección General de Epidemiología; 1994. 11. Secretaría de Educación Pública/Instituto Mexicano de Psiquiatría. Encuesta $\mathrm{N}$ acional sobre el U so de Drogas en la Comunidad Escolar. México, DF: SEP/IMP; 1991.

12. Secretaría de Salud. Encuesta N acional de Adicciones 1998. Alcohol, tabaco y drogas. México, D.F.: D irección General de Epidemiología; agosto 1999. Disponible en: http://www.cmact.com/ceca/estad.htm 13. Maya MA, García G. Estudio epidemiológico sobre el uso de alcohol en población joven de 14 a 18 años. Salud Publica Mex 1986;28:371-379. 14. Grant BF. Prevalence and correlates of alcohol use and DSM-IV alcohol dependence in the U nited States: Results of the national longitudinal alcohol epidemiologic survey. J Stud A Icohol 1997;58: 464-473.

15.Villatoro JA, Medina-Mora ME, C ardiel H, Fleiz C ,Alcántar E, Hernández $\mathrm{S}$ et al. La situación del consumo de sustancias entre estudiantes de la ciudad de México: Medición otoño de 1997. Salud Mental 1999;22:18-30.

16. Anthony JC,W arner LA, Kessler RC. Comparative epidemiology of dependence on tobacco, alcohol, controlled substances, and inhalants. Basic findings from the $\mathrm{N}$ ational Comorbidity Survey. Exp C lin Psychopharmacology 1994;2:244-268.
17. Rojas GE, Medina-Mora ME,Villatoro J, Juárez F, C arreño S, Berenzon $S$. Evolución del consumo de drogas entre estudiantes del $D$ istrito Federal. Salud Mental 1998:21:37-42.

18. Rojas GE, Medina-Mora ME, Juárez F, C arreño S,Villatoro J, Berenzon $\mathrm{S}$, López E. El consumo de bebidas alcohólicas y variables asociadas entre los estudiantes de México. Salud Mental 1995;18:22-27. 19. D urant RH, Smith JA, Kreiter SR, Krowchuck D P.The relationship between early age of onset of initial substance use and engaging in multiple health risk behaviors among young adolescents. Arch Pediatr Adolesc Med 1999;153:286-291.

20. Mora-Ríos J, N atera G. Expectativas, consumo de alcohol y problemas asociados en estudiantes universitarios de la Ciudad de México. Salud Publica Mex 2001:43:89-96.

21.Anthony JC, Petronis KR. Early-onset drug use and risk of later drug problems. Drug Alcohol Depend 1995;40:9-15.

22. Castro MA. Indicadores de riesgo para el consumo problemático de drogas en jóvenes estudiantes. A plicaciones en investigación y atención primaria dentro del plantel escolar. Salud Publica Mex 1990;32:298-308. 23. Kandel D B. Stages in adolescent involvement in drug use. Science $1975 ; 190: 912-914$

24. Kandel DB, Yamaguchi K, Chen K. Stages of progression in drug involvement from adolescence to adulthood: Further evidence for the gateway theory. Stud Alcohol 1992;53: 447-457.

25. Robins LN . Estimating addiction rates and locating target populations: How decomposition into stages helps. En: Rittenhouse JD, ed.The epidemiology of heroin and other narcotics. NIDA Research Monograph 16. Rockville; (MD): N ational Institute on D rug A buse; 1977:25-39.

26.W illner P.A view through the gateway: Expectancies as a possible pathway from alcohol to cannabis. Addiction 2001;96:691-703.

27. N eumark YD, D elva J,Anthony JC. The epidemiology of adolescent inhalant drug involvement. Arch Pediatr Adolesc Med 1998;152:781-786. 28. N ordstrom BL, Kinnunen T, Utman CH, Krall EA, Vokonass PS, Garvey AJ. Predictors of continued smoking over 25 years of follow-up in the normative aging study. Am J Public Health 2000;90(3):404-406. 29. Ray $\mathrm{O}, \mathrm{K}$ sir $\mathrm{CH}$. D rugs, society and human behavior. 7a Ed. Mosby; 1996.

30. Medina-Mora Icaza ME,Villatoro JA, López EK, Berenzon S, C arreño $S$, Juárez F. Los factores que se relacionan con el inicio, el uso continuado y el abuso de sustancias psicoactivas en adolescentes mexicanos. Gac Med Mex 1995;131:383-393.

31. Secretaría de Salud. El consumo de drogas en México: diagnóstico, tendencias y acciones. México, DF: SSA; 1999.

32. Tapia CR, Cravioto $P$, Revuelta A, Kuri P. Los retos de la transición. Adicciones: una dosis de prevención. México, DF: SSA; 1994. (Cuadernos de Salud 5).

33. N azar A, Tapia R, Villa A, León G, Medina-Mora ME, Salvatierra B. Factores asociados al consumo de drogas en adolescentes de áreas urbanas de México. Salud Publica Mex 1994;36: 646-654.

34. Rojas GE, Castro MA, De la Serna J, García G. Análisis regional sobre el uso de drogas en la población estudiantil de México. Salud Publica Mex 1987;29:331-344.

35. Castro SM, Maya MA,A guilar MA. Consumo de sustancias tóxicas y tabaco entre la población estudiantil de 14 a 18 años del D istrito Federal y Zona Metropolitana; medición transversal, 1980. Salud Publica Mex 1982;24:565-574.

36. Secretaría de Salud. Encuesta N acional de Adicciones 1993. D rogas. México, DF:SSA-Dirección General de Epidemiología; 1994.

37. González-Villalpando C, Stern MP,A Arredondo-Pérez B, Mitchell B, Valdez R, Haffner S. Consumo de tabaco en la Ciudad de México. Salud Publica Mex 1994;36:46-50.

38. Unikel C , G alván J, Soriano A, Villatoro J, 0 rtiz A, Rodríguez E. Evolución del consumo de cocaína en México y su presencia entre las clases menos favorecidas. Salud Mental 1998;21:29-36. 
39. Medina-Mora Icaza ME, De la Parra CA, Terroba G G. El consumo de alcohol en la población del distrito federal. Salud Publica Mex 1980; 22(3):281-288.

40. Rojas GE, Fleiz-Bautista C, Medina-Mora ME, Morón MA, Doménech-Rodríguez M. Consumo de alcohol y drogas en estudiantes de Pachuca, Hidalgo. Salud Publica Mex 1999;41(4):297-308.

41. Lazcano-Ponce EC, Hernández B, Cruz-Valdez A, Allen B, Díaz R, Hernández $\mathrm{C}$ et al. $C$ hronic disease risk factors among healthy adolescents attending public schools in the State of Morelos, Mexico. Arch Med Res 2003;34:222-236.

42. Smart RG, Hughes PH, Johnston LD, A numonye A, Khant U, MedinaMora ME et al. A methodology for student drug use surveys. G inebra: W HO ; 1980. (W HO offset Publication N - 50: 5-55).

43. Medina-Mora Icaza ME, Gómez F, C ampillo C.Validity and reliability of a high school drug use questionnaire among Mexican students. Bull $N$ arc 1981;33:67-76

44. Kandel DB, Logan JA. Patterns of drug use from adolescence to young adulthood. I. Periods of risk for initiation, stabilization and decline in use. Am J Public Health 1984; 74:660-666.

45. Medina-Mora Icaza ME. Los conceptos de uso, abuso, dependencia y su definición. En: Roberto Tapia Conyer, ed. Las adicciones, dimensión, impacto y perspectivas. México, DF: Ed. Manual Moderno; 1994:25-55. 46. Indices de marginación municipal del estado de Morelos. Indices de Marginación 2000. México, D F: Consejo N acional de Población, 2000. 47. Rabe-H esketh S, Everitt B.A Handbook of statistical analyses using Stata. Florida-USA: Chapman \& Hall/CRC; 2000: 170-176.

48. Jolliffe IT. Principal components analysis. Springer Series in Statistics. England, 1986.

49.W agner FA, Anthony JC. Into the world of illegal drug use: Exposure opportunity and other mechanisms linking alco hol, to bacco, marijuana, and cocaine use. Am J Epidemiol 2002;155:918-925.

50.W ilcox HC, W agner FA,Anthony JC. Exposure opportunity as a mechanism linking youth marijuana use to hallucinogen use. D rug Alcohol Depend 2002; 66: 127-135

51. Caraveo-Anduaga JJ, C olmenares-Bermúdez E, Saldívar-Hernández GJ. Diferencias por género en el consumo de alcohol en la Ciudad de México. Salud Publica Mex 1999;41:177-188.

52. D ewit DJ,Adlaf EM, 0 fford DR, 0 gborne AC.Age at first alcohol use: A risk factor for the development of alcohol disorders. Am J Psychiatry 2000; 157: 745-750.

53.W agner FA,Anthony JC. From first use to drug dependence: developmental periods of risk for dependence upon marijuana, cocaine, and alcohol. N europsychopharmacology 2002;26: 479-488.

54. Grant BF, Dawson DA. Age at onset of alcohol use and its association with DSM-IV alcohol abuse and dependence: Results from the N ational Longitudinal Alcohol Epidemiologic Survey.J Subst Abuse 1997; 9:103-110

55. Cox DR. Regression models and life tables. JR Stat Soc 1972; 34: 187-222.

56. Breslow N E, Day N E. Statistical methods in cancer research.Volume II.The design and analysis of cohort studies. 0 xford, UK: 0 xford University Press; 1989

57. Singer JD,W illet JB. Designing and analyzing studies of onset, cessation, and relapse: U sing survival analysis in drug abuse prevention research. N IDA Res Monog 1994;142:196-263.

58. Singer JD,W illet JB. It's about time: U sing discrete-time survival analysis to study duration and the timing of events. J Educational Statistics 1993:18:155-195.

59.W illet JB, Singer JD. Investigating onset, cessation, relapse and recovery:W hy you should, and how you can, use discrete-time survival analysis to examine event occurrence.J Consult Clin Psychol 1993; 61: 952-965.

60. Stata C orp. Stata Statistical Software. Release 6.0. Reference Manual. Stata Press, College Station TX, 1999

61. Bobashev GV,Anthony JC. Use of alternating logistic regression in studies of drug-use clustering. Subst Use Misuse 2000;35:1051-1073. 62. Medina-Mora ME, Peña-Corona MP, Cravioto P,Villatoro J, Kuri P. Del tabaco al uso de otras drogas: iel uso temprano de tabaco aumenta la probabilidad de usar otras drogas? Salud Publica Mex 2002;44 supl 1:S109-S115.

63.Van Etten MLO, N eumark YD,Anthony JC. Initial opportunity to use marijuana and the transition to first use: United States, 1979-1994. D rug Alcohol Depend 1997;49:1-7.

64.Van Etten ML,Anthony JC. Comparative epidemiology of initial drug opportunities and transitions to first use: Marijuana, cocaine, hallucinogens and heroin. DrugAlcohol Depend 1999;54:117-125. 65.Van Etten ML, N eumark YD,Anthony JC. Male-female differences in the earliest stages of drug involvement. Addiction 1999;94:1413-1419. 66. D elva J,Van Etten ML, González GB, C edeno MA, Penna M, C aris LH, Anthony JC. First opportunities to try drugs and the transition to first drug use: Evidence from a national school survey in Panama. Subst Use Misuse 1999;34:1451-1467.

67. Wagner FA, Borges $G$, Medina-Mora ME, Benjet C, Villatoro JA. Parental involvement, needs and drug-related service utilization among Mexican middle and high school students. J Family Social W ork 2001:6:79-95.

68. 0 'Malley PM, Bachman JG, Johnston LD. Reliability and consistency in self-reports of drug use. Int J Addict 1983;18:805-824.

69. Johnson TP, Mott JA.The reliability of self-reported age of onset of to bacco, alcohol and illicit drug use. Addiction 2001;96:1187-1198. 